\title{
HEREDITARY PROPERTIES OF CHARACTER INJECTIVITY WITH APPLICATIONS TO SEMIGROUP ALGEBRAS
}

\author{
M. ESSMAILI*, M. FOZOUNI AND J. LAALI
}

\begin{abstract}
In this paper, we investigate the notion $\phi$-injectivity for Banach $A$-modules, where $\phi$ is a character on $A$. We obtain some hereditary properties of $\phi$-injectivity for certain classes of Banach modules related to closed ideals. These results allow us to study $\phi$-injectivity of certain Banach $A$-modules in commutative case, specially $\ell^{1}$-semilattice algebras. As an application, we give an example of a non-injective Banach module which is $\phi$-injective for each character $\phi$.
\end{abstract}

\section{INTRODUCTION}

Suppose that $A$ is a Banach algebra. We denote by $\mathbf{A}-\bmod$ and $\bmod -\mathbf{A}$ the categories of Banach left $A$-modules and Banach right $A$-modules, respectively. In the case where $A$ is unital, we also denote by $\mathbf{A}$-unmod the categories of unital Banach left $A$-modules. For each $E, F \in \mathbf{A}$-mod, let ${ }_{A} B(E, F)$ be the closed subspace of $B(E, F)$ consisting of the left $A$-module morphisms. An operator $T \in B(E, F)$ is called admissible if $\operatorname{ker} T$ and $\operatorname{Im} T$ are closed complemented subspaces of $E$ and $F$, respectively. It is easy to verify that $T$ is admissible if and only if there exists $S \in B(F, E)$ such that $T \circ S \circ T=T$.

A Banach left $A$-module $E$ is called injective if for each $F, K \in \mathbf{A - m o d}$ and admissible monomorphism $T \in \in_{A} B(F, K)$, the induced map ${ }_{A} B(K, E) \longrightarrow_{A} B(F, E)$ is onto. We also say $E \in \bmod -\mathbf{A}$ is flat if the dual module of $E^{*} \in \mathbf{A}$-mod is injective with the following left module action:

$$
(a \cdot f)(x)=f(x \cdot a) \quad(a \in A, x \in E) .
$$

The notions of injectivity and flatness of Banach algebras were introduced by A. Ya. Helemskii. These notions have been studied for various classes of Banach modules; see [3], [6], [11] and [13] for more details. Recently, Ramsden in [11] studied injectivity and flatness of Banach modules over semigroup algebras. It is well known that if $A$ is amenable, then every Banach $A$-modules is flat but the converse is a long standing open problem. We recall that the answer is positive for some classes of Banach algebras associated with locally compact groups such as, the class of group algebras and measure algebras; see [3] and [12].

Kaniuth, Lau and Pym introduced and studied in [7] and [8] the notion of $\phi$-amenability for Banach algebras, where $\phi: A \longrightarrow \mathbb{C}$ is a character, i.e., a nonzero homomorphism on $A$. Afterwards, Monfared introduced and studied in [9] the notion of character amenability for Banach algebras. Let $\Delta(A)$ be the set of

2010 Mathematics Subject Classification. Primary 46M10 Secondary 43A20, 46H25.

Key words and phrases. Injectivity, $\phi$-injectivity, $\phi$-amenability, semigroup algebras. 
all characters of the Banach algebra $A$, and let $\phi \in \Delta(A)$. The Banach algebra $A$ is called left $\phi$-amenable if for all Banach $A$-bimodules $E$ for which the left module action is given by

$$
a \cdot x=\phi(a) x \quad(a \in A, x \in E),
$$

every derivation $D: A \longrightarrow E^{*}$ is inner. It is clear that amenability of $A$ implies $\phi$-amenability for all $\phi \in \Delta(A)$.

Recently, Nasr-Isfahani and Soltani Renani in [10] introduced and studied the notion of $\phi$-injectivity and $\phi$-flatness for Banach modules (see Definition 2.1). As an important result, it is shown in [10, Proposition 3.1] that the Banach algebra $A$ is left $\phi$-amenable if and only if every Banach left $A$-modules $E$ is $\phi$-flat. Indeed, this result gives a positive answer to the above open problem arises by $\mathrm{A}$. Ya. Helemskii in this homology setting based on character $\phi$. Furthermore, they obtained some necessary and sufficient conditions for $\phi$-injectivity and characterized $\phi$-injectivity of Banach modules in terms of a coretraction problem; see [10, Theorem 2.4].

This paper is organized as follows. In Section 2, after recalling some definitions, we investigate some properties of $\phi$-injectivity for Banach modules. Indeed, we obtain a sufficient condition for $\phi$-injectivity of Banach left $A$-modules, in the case where $A$ is a commutative Banach algebra. Moreover, we give some hereditary properties of $\phi$-injectivity for Banach $A$-modules related to the closed ideals of Banach algebra $A$. As the main result, we show that if $J$ is a left invariant complemented ideal in $A$, then $\phi$-injectivity of $J$ and $A / J$ in A-mod is equivalent to the $\phi$-injectivity of $A$ in $\mathbf{A}$-mod (Theorem 2.10). In Section 3, by using the results of Section 2, we study $\phi$-injectivity of certain $\ell^{1}$-semilattice algebras and show that $\ell^{1}\left(\mathbb{N}_{\wedge}\right)$ as a Banach left $\ell^{1}\left(\mathbb{N}_{\wedge}\right)$-module is $\phi$-injective for each character $\phi$, although is not injective.

\section{2. $\phi$-INJECTIVITY AND SOME HEREDITARY PROPERTIES}

First, we recall some standard notations that we shall use and define the notions of $\phi$-injectivity and $\phi$-flatness of Banach modules.

Let $A$ be a Banach algebra and $E \in \mathbf{A}$-mod. Throughout the paper, we regard $E$ as a Banach left $A^{\sharp}$-module (the unitization of $A$ ) with the following left module action:

$$
(a, \lambda) \cdot x=a \cdot x+\lambda x \quad(a \in A, \lambda \in \mathbb{C}, x \in E) .
$$

Moreover, the space $B(A, E)$ is a Banach $A$-bimodule with the following module actions:

$$
(a \cdot T)(b)=T(b a), \quad(T \cdot a)(b)=T(a b) \quad(T \in B(A, E), a, b \in A) .
$$

Suppose that $A$ is a Banach algebra and $\phi \in \Delta(A)$. For each $E \in \mathbf{A}-\bmod$ we define,

$$
I(\phi, E)=\operatorname{span}\{a \cdot x-\phi(a) x: a \in A, x \in E\} .
$$

Following [10], we also consider

${ }_{\phi} B\left(A^{\sharp}, E\right)=\left\{T \in B\left(A^{\sharp}, E\right): T(a b-\phi(b) a)=a \cdot T\left(b-\phi(b) e^{\sharp}\right)\right.$ for all $\left.a, b \in A\right\}$, 
where $e^{\sharp}=(0,1)$ denotes the unite of $A^{\sharp}$. It is straightforward to check that ${ }_{\phi} B\left(A^{\sharp}, E\right)$ is a closed $A$-submodule of $B\left(A^{\sharp}, E\right)$. Moreover, we define the canonical morphism $_{\phi} \Pi^{\sharp}: E \longrightarrow{ }_{\phi} B\left(A^{\sharp}, E\right)$ as follows:

$$
{ }_{\phi} \Pi^{\sharp}(x)(a)=a \cdot x \quad\left(x \in E, a \in A^{\sharp}\right) .
$$

Definition 2.1. Let $A$ be a Banach algebra, $\phi \in \Delta(A)$ and $E \in \mathbf{A}$-mod. We say that $E$ is $\phi$-injective if, for each $F, K \in \mathbf{A}$-mod and admissible monomorphism $T: F \longrightarrow K$ with $I(\phi, K) \subseteq \operatorname{Im}(T)$, the induced map $T_{E}:{ }_{A} B(K, E) \longrightarrow$ ${ }_{A} B(F, E)$ defined by $T_{E}(R)=R \circ T$ is onto.

The following theorem gives a characterization of $\phi$-injectivity in terms of a coretraction problem.

Theorem 2.2. ([10, Theorem 2.4]) Let $A$ be a Banach algebra and $\phi \in \Delta(A)$. For $E \in \boldsymbol{A}$-mod the following statements are equivalent.

(i) $E$ is $\phi$-injective.

(ii) ${ }_{\phi} \Pi^{\sharp} \in{ }_{A} B\left(E,{ }_{\phi} B\left(A^{\sharp}, E\right)\right)$ is a coretraction, (that is there exists ${ }_{\phi} \rho^{\sharp} \in$ ${ }_{A} B\left({ }_{\phi} B\left(A^{\sharp}, E\right), E\right)$ such that is a left inverse for $\left.{ }_{\phi} \Pi^{\sharp}\right)$.

A Banach right (left) $A$-module $E$ is $\phi$-flat if $E^{*}$ is $\phi$-injective as a left (right) $A$-module. It is shown that Banach algebra $A$ is left $\phi$-amenable if and only if each Banach left $A$-module $E$ is $\phi$-flat [10, Proposition 3.1].

In this section, we give some hereditary properties of $\phi$-injectivity for certain classes of Banach modules. We also consider some hereditary properties of $\phi$ injectivity of Banach left $A$-modules with their ideals. We first give a sufficient condition for $\phi$-injectivity of Banach left $A$-module $E$ in the case where $A$ is a commutative Banach algebra. Following [1, Definition 1.4.4], the annihilator of $E$ is defined by $E^{\perp}=\{a \in A: a \cdot E=\{0\}\}$.

Theorem 2.3. Let $A$ be a commutative Banach algebra, $\phi \in \Delta(A)$ and $E \in$ $\boldsymbol{A}$-mod. If $E^{\perp} \cap(\operatorname{ker}(\phi))^{c} \neq \emptyset$, then $E$ is $\phi$-injective.

Proof. Let $a_{0} \in E^{\perp} \cap(\operatorname{ker}(\phi))^{c}$. We can assume that $\phi\left(a_{0}\right)=1$ and define the $\operatorname{map}_{\phi} \rho^{\sharp}:_{\phi} B\left(A^{\sharp}, E\right) \longrightarrow E$ by

$$
{ }_{\phi} \rho^{\sharp}(T)=T\left(e^{\sharp}-a_{0}\right) \quad\left(T \in_{\phi} B\left(A^{\sharp}, E\right)\right) .
$$

Hence, for each $x \in E$ we have

$$
{ }_{\phi} \rho^{\sharp} \circ_{\phi} \Pi^{\sharp}(x)={ }_{\phi} \Pi^{\sharp}(x)\left(e^{\sharp}-a_{0}\right)=\left(e^{\sharp}-a_{0}\right) \cdot x=x .
$$

Therefore, ${ }_{\phi} \rho^{\sharp} \circ_{\phi} \Pi^{\sharp}=I_{E}$. On the other hand, for each $a \in A$ and $T \in_{\phi} B\left(A^{\sharp}, E\right)$ we have

$$
\begin{aligned}
{ }_{\phi} \rho^{\sharp}(a \cdot T) & =(a \cdot T)\left(e^{\sharp}-a_{0}\right)=T\left(\left(e^{\sharp}-a_{0}\right) \cdot a\right) \\
& =T\left(a-a_{0} a\right) \\
& =T\left(a \phi\left(a_{0}\right)-a a_{0}\right) .
\end{aligned}
$$

Since $T \in_{\phi} B\left(A^{\sharp}, E\right)$ we have $T\left(a \phi\left(a_{0}\right)-a a_{0}\right)=a \cdot T\left(e^{\sharp}-a_{0}\right)$. Now, using (2.1) we conclude that

$$
{ }_{\phi} \rho^{\sharp}(a \cdot T)=a \cdot T\left(e^{\sharp}-a_{0}\right)=a \cdot{ }_{\phi} \rho^{\sharp}(T) .
$$


It follows that ${ }_{\phi} \rho^{\sharp}$ is a left $A$-module morphism. Hence, $E$ is a $\phi$-injective Banach left $A$-module.

Corollary 2.4. Let $A$ be a commutative Banach algebra, and $J$ be a closed ideal of $A$ such that $\phi_{\mid J} \neq 0$. Then $A / J$ is $\phi$-injective as a Banach left A-module.

Proof. Since $\phi_{\mid J} \neq 0$, it is easy to check that $(A / J)^{\perp} \cap(\operatorname{ker}(\phi))^{c} \neq \emptyset$. Now, apply Theorem 2.3.

Corollary 2.5. Let $A$ be a commutative Banach algebra, $\phi \in \Delta(A)$ and let $E \in \boldsymbol{A}$-mod with $I(\phi, E)=\{0\}$. Then for all $\psi \in \Delta(A) \backslash\{\phi\}, E \in \boldsymbol{A}$ - $\boldsymbol{m o d}$ is $\psi$-injective.

Proof. Since $\phi \neq \psi$ there exists $a_{0} \in A$ such that $\phi\left(a_{0}\right)=0$ and $\psi\left(a_{0}\right)=1$. On the other hand, since $I(\phi, E)=\{0\}$ we conclude that $a_{0} \in E^{\perp} \cap(\operatorname{ker}(\psi))^{c}$ and the proof is complete.

Now, we give some hereditary properties of $\phi$-injectivity of Banach modules that we shall use. Recall that $E \in \mathbf{A}$-mod is faithful if $A \cdot x=\{0\}$ implies that $x=0$.

Theorem 2.6. Let $A$ be a Banach algebra, $E \in \boldsymbol{A}$-mod, $\phi \in \Delta(A)$ and $J$ be a closed ideal of $A$ such that $\phi_{\mid J} \neq 0$.

(i) Suppose that $J$ has an identity and $E \in \boldsymbol{J}$-unmod. If $E \in \boldsymbol{A}$-mod is $\phi$-injective, then $E \in \boldsymbol{J}$-unmod is $\phi_{\mid J}$-injective.

(ii) If $E \in \boldsymbol{J}$-mod is $\phi_{\mid J}$-injective and faithful, then $E \in \boldsymbol{A}$-mod is $\phi$ injective.

Proof. (i) Suppose that $E \in \mathbf{A}$-mod is $\phi$-injective. Let $F$ and $K$ be in $\mathbf{J}$-mod and $T: F \longrightarrow K$ be an admissible monomorphism with $I\left(\phi_{\mid J}, K\right) \subseteq \operatorname{Im} T$. We claim that the induced map, ${ }_{J} B(K, E) \longrightarrow{ }_{J} B(F, E)$ defined by, $R \longrightarrow R \circ T$ is onto. Suppose that $e_{J}$ is the identity of $J$. We can consider $F$ and $K$ as Banach left $A$-modules with the following module actions:

$$
\begin{array}{ll}
a \bullet f=\left(a e_{J}\right) \cdot f & (a \in A, f \in F), \\
a \bullet k=\left(a e_{J}\right) \cdot k & (a \in A, k \in K) .
\end{array}
$$

We denote these $A$-modules with $\widetilde{F}$ and $\widetilde{K}$, respectively. Take $W \in_{J} B(F, E)$ and define the map $\widetilde{W}: \widetilde{F} \longrightarrow E$ by $\widetilde{W}(f)=W(f)$. For each $a \in A$ and $f \in F$ we have,

$$
\begin{aligned}
\widetilde{W}(a \bullet f) & =W\left(\left(a e_{J}\right) \cdot f\right)=\left(a e_{J}\right) \cdot W(f) \\
& =a \cdot\left(e_{J} \cdot W(f)\right)=a \cdot W(f) \\
& =a \cdot \widetilde{W}(f) .
\end{aligned}
$$


So $\widetilde{W}$ is a left $A$-module morphism. Moreover, the map $\widetilde{T}: \widetilde{F} \longrightarrow \widetilde{K}$ defined by $\widetilde{T}(f)=T(f)$ is an admissible monomorphism such that

$$
\begin{aligned}
I(\phi, \widetilde{K}) & =\operatorname{span}\{a \bullet k-\phi(a) k: a \in A, k \in K\} \\
& =\operatorname{span}\left\{\left(a e_{J}\right) \cdot k-\phi\left(a e_{J}\right) k: a \in A, k \in K\right\} \\
& \subseteq \operatorname{Im} T=\operatorname{Im} \widetilde{T} .
\end{aligned}
$$

Since $E \in \mathbf{A}$-mod is $\phi$-injective, there exist $S \in_{A} B(\widetilde{K}, E)$ such that $S \circ \widetilde{T}=\widetilde{W}$. On the other hand, for each $a \in J$ and $k \in K$ we have

$$
a \cdot S(k)=S(a \bullet k)=S\left(\left(a e_{J}\right) \cdot k\right)=S(a \cdot k) .
$$

It follows that $S \in{ }_{J} B(K, E)$. Now, we conclude that $E \in \mathbf{J}$-unmod is $\phi_{\mid J^{-}}$ injective.

(ii) Let $F$ and $K$ be in $\mathbf{A}$-mod and $T: F \longrightarrow K$ be an admissible monomorphism and take $W \in{ }_{A} B(F, E)$. So $W \in{ }_{J} B(F, E)$ and there exists $S \in{ }_{J} B(K, E)$ such that $S \circ T=W$. For each $a \in J, b \in A$ and $k \in K$, we have

$$
\begin{aligned}
a \cdot(S(b \cdot k)-b \cdot S(k)) & =a \cdot S(b \cdot k)-(a b) \cdot S(k) \\
& =S(a b \cdot k)-S(a b \cdot k)=0 .
\end{aligned}
$$

Since $E \in \mathbf{J}-\mathbf{m o d}$ is faithful, we conclude that $S(b \cdot k)=b \cdot S(k)$. It follows that $S \in \in_{A} B(K, E)$ and the proof is complete.

Corollary 2.7. Let $A$ be a Banach algebra, $\phi \in \Delta(A)$ and $J$ be a closed ideal of $A$ with an identity such that $\phi_{\mid J} \neq 0$. Then $J \in \boldsymbol{A}$ - $\boldsymbol{m o d}$ is $\phi$-injective if and only if $J \in \boldsymbol{J}$ - $\bmod$ is $\phi_{\mid J}$-injective.

B. E. Forrest in [5] introduced the notion of invariantly complemented submodules in categories of Banach modules. In the sequel, we obtain some results for $\phi$-injectivity of invariantly complemented ideals.

Definition 2.8. ([5, Definition 6.3]) Let $X$ be a Banach left $A$-module and $Y$ be a Banach $A$-submodule of $X$. We say that $Y$ is left (right) invariantly complemented in $X$ if there exists $P \in{ }_{A} B(X, Y)\left(P \in B_{A}(X, Y)\right)$ such that $P^{2}=P$ and $P(X)=Y$.

Theorem 2.9. Let $\left\{E_{\alpha}\right\}_{\alpha \in \Gamma}$ be a collection of Banach left $A$-modules and consider $E=\ell^{1}-\bigoplus_{\alpha \in \Gamma} E_{\alpha}$ as a Banach left A-module with the natural module action.

(i) If $E$ is $\phi$-injective, then for each $\alpha \in \Gamma, E_{\alpha}$ is $\phi$-injective.

(ii) Conversely, if $\Gamma$ is finite and each $E_{\alpha}$ is $\phi$-injective, then $E$ is $\phi$-injective.

Proof. (i) It is obvious that each $E_{\alpha}$ is left invariantly complemented in $E$. Hence, for each $\alpha \in \Gamma$, let $P_{\alpha} \in{ }_{A} B\left(E, E_{\alpha}\right)$ such that $P_{\alpha}(E)=E_{\alpha}$ and $P_{\alpha}^{2}=P_{\alpha}$. Also, let $i_{\alpha}: E_{\alpha} \longrightarrow E$ be the natural embedding of $E_{\alpha}$ into $E$.

Let $E$ be $\phi$-injective. Then there exists ${ }_{\phi} \rho^{E} \in{ }_{A} B\left({ }_{\phi} B\left(A^{\sharp}, E\right), E\right)$ such that is a left inverse for ${ }_{\phi} \Pi^{E}: E \longrightarrow{ }_{\phi} B\left(A^{\sharp}, E\right)$. For each $\alpha \in \Gamma$, we define the map ${ }_{\phi} \rho^{\alpha}:_{\phi} B\left(A^{\sharp}, E_{\alpha}\right) \longrightarrow E_{\alpha}$ by

$$
{ }_{\phi} \rho^{\alpha}(T)=P_{\alpha}{ }_{\phi} \rho^{E}\left(i_{\alpha} \circ T\right) \quad\left(T \in_{\phi} B\left(A^{\sharp}, E_{\alpha}\right)\right) .
$$


We claim that ${ }_{\phi} \rho^{\alpha}$ is a left $A$-module morphism such that ${ }_{\phi} \rho^{\alpha} \circ_{\phi} \Pi^{\alpha}=I_{E_{\alpha}}$. Indeed, since for each $x \in E_{\alpha}, i_{\alpha} \circ_{\phi} \Pi^{\alpha}(x)={ }_{\phi} \Pi^{E}\left(i_{\alpha}(x)\right)$, so we have

$$
\begin{aligned}
{ }_{\phi} \rho^{\alpha} \circ_{\phi} \Pi^{\alpha}(x) & =P_{\alpha} \circ_{\phi} \rho^{E}\left(i_{\alpha} \circ_{\phi} \Pi^{\alpha}(x)\right)=P_{\alpha} \circ_{\phi} \rho^{E}\left({ }_{\phi} \Pi^{E}\left(i_{\alpha}(x)\right)\right) \\
& =P_{\alpha}\left(i_{\alpha}(x)\right)=x .
\end{aligned}
$$

Therefore, ${ }_{\phi} \rho^{\alpha} \circ_{\phi} \Pi^{\alpha}=I_{E_{\alpha}}$. On the other hand, since $P_{\alpha} \in{ }_{A} B\left(E, E_{\alpha}\right)$ it is easy to see that ${ }_{\phi} \rho^{\alpha}$ is a left $A$-module morphism and the proof is complete.

(ii) Suppose that for each $\alpha \in \Gamma, E_{\alpha}$ is $\phi$-injective. So, for each $\alpha \in \Gamma$ there exists ${ }_{\phi} \rho^{\alpha} \in{ }_{A} B\left({ }_{\phi} B\left(A^{\sharp}, E_{\alpha}\right), E_{\alpha}\right)$ for which ${ }_{\phi} \rho^{\alpha} \circ_{\phi} \Pi^{\alpha}=I_{E_{\alpha}}$. Define the map $\rho:_{\phi} B\left(A^{\sharp}, E\right) \longrightarrow E$ by

$$
\rho(T)=\left({ }_{\phi} \rho^{\alpha}\left(P_{\alpha} \circ T\right)\right)_{\alpha \in \Gamma} \quad\left(T \in{ }_{\phi} B\left(A^{\sharp}, E\right)\right) .
$$

Since $\Gamma$ is finite, $\rho$ is well-defined. For each $a \in A$ and $T \in_{\phi} B\left(A^{\sharp}, E\right)$ we have

$$
\begin{aligned}
\rho(a \cdot T) & =\left({ }_{\phi} \rho^{\alpha}\left(P_{\alpha} \circ(a \cdot T)\right)\right)_{\alpha \in \Gamma}=\left({ }_{\phi} \rho^{\alpha}\left(a \cdot\left(P_{\alpha} \circ T\right)\right)\right)_{\alpha \in \Gamma} \\
& =\left(a \cdot{ }_{\phi} \rho^{\alpha}\left(P_{\alpha} \circ T\right)\right)_{\alpha \in \Gamma}=a \cdot\left({ }_{\phi} \rho^{\alpha}\left(P_{\alpha} \circ T\right)\right)_{\alpha \in \Gamma} \\
& =a \cdot \rho(T) .
\end{aligned}
$$

Moreover, if $x=\left(x_{\alpha}\right)_{\alpha \in \Gamma}$ is an arbitrary element of $E$, it is easy to see that $P_{\alpha} \circ_{\phi} \Pi^{E}(x)={ }_{\phi} \Pi^{\alpha}\left(x_{\alpha}\right)$. Hence,

$$
\begin{aligned}
\rho \circ_{\phi} \Pi^{E}(x) & =\left({ }_{\phi} \rho^{\alpha}\left(P_{\alpha} \circ_{\phi} \Pi^{E}(x)\right)\right)_{\alpha \in \Gamma}=\left({ }_{\phi} \rho^{\alpha}\left({ }_{\phi} \Pi^{\alpha}\left(x_{\alpha}\right)\right)\right)_{\alpha \in \Gamma} \\
& =\left(x_{\alpha}\right)_{\alpha \in \Gamma}=x .
\end{aligned}
$$

Therefore, we conclude that $E$ is $\phi$-injective.

Theorem 2.10. Let $A$ be a Banach algebra, $\phi \in \Delta(A), B$ be a subalgebra of $A$ and $J$ be a closed left ideal of $A$. Then the following assertions holds:

(i) If $B$ is left invariantly complemented in $A$ and $A$ is $\phi$-injective in $\boldsymbol{A}$-mod, then $B$ is $\phi$-injective in $\boldsymbol{A}$-mod.

(ii) If $J$ is left invariantly complemented, then $J$ and $A / J$ are $\phi$-injective in $\boldsymbol{A}$-mod if and only if $A$ is $\phi$-injective in $\boldsymbol{A}$-mod.

Proof. (i) Since $B$ is left invariantly complemented in $A$, there exists an onto projection $P \in{ }_{A} B(A, B)$. Hence

$$
A \cong \operatorname{Im} P \oplus \operatorname{ker} P=B \oplus \operatorname{ker} P,
$$

as a Banach left $A$-module. Therefore, by Theorem 2.9 it follows that $B$ is $\phi$ injective in A-mod.

(ii) Since $J$ is a left invariant complemented ideal in $A$, there exists an onto projection $P \in{ }_{A} B(A, J)$. We claim that $A \cong J \oplus \frac{A}{J}$ as a Banach left $A$-module. To see this, define the map $T: A \longrightarrow J \oplus \frac{A}{J}$ by

$$
T(a)=(P(a), a+J) \quad(a \in A) .
$$

First, $T$ is a left $A$-module morphism, because for each $a, b \in A$ we have,

$$
\begin{aligned}
T(a b) & =(P(a b), a b+J)=(a P(b), a(b+J)) \\
& =a \cdot(P(b), b+J)=a \cdot T(b) .
\end{aligned}
$$


On the other hand, if $a \in \operatorname{Im} P \cap \operatorname{ker} P$, then there exists $b \in A$ such that $P(b)=a$. Hence, $a=P(b)=P(P(b))=P(a)=0$. This follows that $\operatorname{Im} P \cap \operatorname{ker} P=\{0\}$ and so $T$ is one-to-one. Moreover, $T$ is onto because for each $(a, b+J) \in J \oplus \frac{A}{J}$ if we put $c=a+b-P(b)$, then $T(c)=(a, b+J)$. Now, the result follows from Theorem 2.9 .

As an application of Theorem 2.10 and Corollary 2.4, we have the following result for commutative Banach algebras.

Corollary 2.11. Let $A$ be a commutative Banach algebra, $\phi \in \Delta(A)$ and let $J$ be a closed invariant complemented ideal of $A$ such that $\phi_{\mid J} \neq 0$. Then $A \in \boldsymbol{A}$ - mod is $\phi$-injective if and only if $J \in \boldsymbol{A}$ - $\boldsymbol{m o d}$ is $\phi$-injective.

\section{Applications to semigroup algebras}

In this section, we apply our later results to study $\phi$-injectivity of certain commutative semigroup algebras and give some examples of non-injective modules which are $\phi$-injective for each character $\phi$. First, we need some basic facts about semigroup algebras.

Let $S$ be a semigroup and let $E(S)=\left\{s \in S: s^{2}=s\right\}$. We say that $S$ is a semilattice if $S$ is commutative and $E(S)=S$. A semi-character on $S$ is a nonzero homomorphism $\widehat{\phi}: S \longrightarrow\{z \in \mathbb{C}:|z| \leq 1\}$. The space of semi-characters on $S$ is denoted by $\Phi_{S}$. The semi-character $\widehat{\phi}_{S}: S \longrightarrow\{z \in \mathbb{C}:|z| \leq 1\}$, defined by

$$
\widehat{\phi}_{S}(t)=1 \quad(t \in S)
$$

is called the augmentation character on $S$. For each $\widehat{\phi} \in \Phi_{S}$, we associate the $\operatorname{map} \phi: \ell^{1}(S) \longrightarrow \mathbb{C}$ defined by

$$
\phi(f)=\sum_{s \in S} \widehat{\phi}(s) f(s) \quad\left(f \in \ell^{1}(S)\right) .
$$

It is easy to verify that $\phi \in \Delta\left(\ell^{1}(S)\right)$ and every character on $\ell^{1}(S)$ arises in this way. Indeed, we have

$$
\Delta\left(\ell^{1}(S)\right)=\left\{\phi: \widehat{\phi} \in \Phi_{S}\right\}
$$

We also define the convolution of two elements $f, g \in \ell^{1}(S)$ by

$$
(f * g)(s)=\sum_{u v=s} f(u) g(v) \quad(s \in S),
$$

where $\sum_{u v=s} f(u) g(v)=0$, when there are no elements $u, v \in S$ with $u v=s$. Then $\left(\ell^{1}(S), *,\|\cdot\|_{1}\right)$ becomes a Banach algebra that is called the semigroup algebra of $S$. The following proposition immediately follows from Corollary 2.11.

Proposition 3.1. Let $S$ be a semilattice, $\phi \in \Delta\left(\ell^{1}(S)\right)$ and I be a closed invariant complemented ideal in $\ell^{1}(S)$ such that $\phi_{\left.\right|_{I}} \neq 0$. Then $\ell^{1}(S) \in \ell^{1}(S)-\bmod$ is $\phi$ injective if and only if $I \in \ell^{1}(S)$ - $\bmod$ is $\phi$-injective.

Let $\ell^{1}\left(\mathbb{N}_{\wedge}\right)$ be the semigroup algebra on semigroup $S=(\mathbb{N}, \wedge)$ with the following product:

$$
\mathbb{N} \times \mathbb{N} \longrightarrow \mathbb{N}, \quad(m, n) \longrightarrow m \wedge n=\min \{m, n\}
$$


It is easy to check that $\Phi_{S}=\left\{\widehat{\phi}_{n}: n \in \mathbb{N}\right\}$, where for each $n \in \mathbb{N}$,

$$
\widehat{\phi}_{n}(m)=\left\{\begin{array}{ll}
1 & \text { if } \quad m \geq n \\
0 & \text { if } \quad m<n
\end{array} \quad(m \in \mathbb{N}) .\right.
$$

For each $n \in \mathbb{N}$, let $I_{n}$ be the ideal of $\ell^{1}\left(\mathbb{N}_{\wedge}\right)$ generated by the set $\left\{\delta_{1}, \delta_{2}, \delta_{3}, \ldots, \delta_{n}\right\}$. It is easy to see that $\ell^{1}\left(\mathbb{N}_{\wedge}\right) / I_{n}$ does not have an identity. So $I_{n}$ does not have a modular identity and using [12, Corollary 2.2 .8 (ii)], we conclude that $\ell^{1}\left(\mathbb{N}_{\wedge}\right) / I_{n}$ is not injective as a Banach left $\ell^{1}\left(\mathbb{N}_{\wedge}\right)$-module. Furthermore, we recall that $\ell^{1}\left(\mathbb{N}_{\wedge}\right)$ as a Banach left $\ell^{1}\left(\mathbb{N}_{\wedge}\right)$-module is not injective, because it does not have a right identity [2, Example 4.10]. As mentioned above, we regard the map $\phi_{n}: \ell^{1}\left(\mathbb{N}_{\wedge}\right) \longrightarrow \mathbb{C}$ as a character on $\ell^{1}\left(\mathbb{N}_{\wedge}\right)$ which is defined by

$$
\phi_{n}(f)=\sum_{i=n}^{\infty} f(i) \quad\left(f \in \ell^{1}\left(\mathbb{N}_{\wedge}\right)\right)
$$

The following theorem shows that $\ell^{1}\left(\mathbb{N}_{\wedge}\right)$ as a left $\ell^{1}\left(\mathbb{N}_{\wedge}\right)$-module is $\phi$-injective for each $\phi \in \Delta\left(\ell^{1}\left(\mathbb{N}_{\wedge}\right)\right)$, although is not injective.

Theorem 3.2. With the above notations, we have following assertions:

(i) $\ell^{1}\left(\mathbb{N}_{\wedge}\right) / I_{n}$ as a Banach left $\ell^{1}\left(\mathbb{N}_{\wedge}\right)$-module is $\phi_{n}$-injective, for each $n \in \mathbb{N}$.

(ii) $\ell^{1}\left(\mathbb{N}_{\wedge}\right)$ as a Banach left $\ell^{1}\left(\mathbb{N}_{\wedge}\right)$-module is $\phi_{n}$-injective, for each $n \in \mathbb{N}$.

Proof. (i) Since $\ell^{1}\left(\mathbb{N}_{\wedge}\right)$ is commutative and $\left(\phi_{n}\right)_{\mid I_{n}} \neq 0$, by Corollary 2.4, it follows that $\ell^{1}\left(\mathbb{N}_{\wedge}\right) / I_{n}$ as a Banach left $\ell^{1}\left(\mathbb{N}_{\wedge}\right)$-module is $\phi_{n}$-injective.

(ii) First, we show that for each $n \in \mathbb{N}, I_{n}$ is an invariant complemented ideal of $\ell^{1}\left(\mathbb{N}_{\wedge}\right)$. To see this, suppose that the map $P_{n}: \ell^{1}\left(\mathbb{N}_{\wedge}\right) \longrightarrow I_{n}$ is defined by

$$
P_{n}(f)=\sum_{i=1}^{n-1} f(i) \delta_{i}+\left(\sum_{i=n}^{\infty} f(i)\right) \delta_{n} \quad\left(f \in \ell^{1}\left(\mathbb{N}_{\wedge}\right)\right) .
$$

It is easy to check that $P_{n}$ is a projection on $I_{n}$. Moreover, if $f$ or $g$ belong to $I_{n}$ we have $P_{n}(f * g)=f * P_{n}(g)$. Now suppose that $f, g$ are not in $I_{n}$. We can suppose that $f=\delta_{i}$ and $g=\delta_{j}$ such that $n<i \leq j$. Hence, we have

$$
P_{n}\left(\delta_{i} * \delta_{j}\right)=P_{n}\left(\delta_{i}\right)=\delta_{n}=\delta_{i} * \delta_{n}=\delta_{i} * P_{n}\left(\delta_{j}\right) .
$$

This follows that $I_{n}$ is an invariant complemented closed ideal of $\ell^{1}\left(\mathbb{N}_{\wedge}\right)$.

Case 1: We prove that $A=\ell^{1}\left(\mathbb{N}_{\wedge}\right)$ is $\phi_{1}$-injective. Since $I_{1}$ is invariantly complemented in $A,\left(\phi_{1}\right)_{I_{1}} \neq 0$ and $A$ is commutative, by Proposition 3.1, it suffices to show that $I_{1} \in \mathbf{A}$-mod is $\phi_{1}$-injective.

Define the map $\rho:{ }_{\phi_{1}} B\left(A^{\sharp}, I_{1}\right) \longrightarrow I_{1}$ by

$$
\rho(T)=T\left(e^{\sharp}\right) \quad\left(T \in{ }_{\phi_{1}} B\left(A^{\sharp}, I_{1}\right) .\right.
$$

Clearly $\rho$ is a left inverse for ${ }_{\phi_{1}} \Pi^{\sharp}: I_{1} \longrightarrow{ }_{\phi_{1}} B\left(A^{\sharp}, I_{1}\right)$. We claim that $\rho$ is a left A-module morphism.

For each $f \in A$ and $g \in I_{1}$ we note that $f * g=\phi_{1}(f) g$. Hence, if $T \in$ ${ }_{\phi_{1}} B\left(A^{\sharp}, I_{1}\right)$, then for each $f, g \in A$ we have

$$
T\left(f * g-\phi_{1}(g) f\right)=f \cdot T\left(g-\phi_{1}(g) e^{\sharp}\right) .
$$


In the case where $g=\delta_{1}$, we conclude that

$$
\begin{aligned}
T\left(\phi_{1}(f) \delta_{1}-\phi_{1}(g) f\right) & =T\left(f * g-\phi_{1}(g) f\right) \\
& =f \cdot T\left(g-\phi_{1}(g) e^{\sharp}\right) \\
& =f * T\left(g-\phi_{1}(g) e^{\sharp}\right) \\
& =\phi_{1}(f) T\left(\delta_{1}-\phi_{1}\left(\delta_{1}\right) e^{\sharp}\right) \\
& =\phi_{1}(f) T\left(\delta_{1}-e^{\sharp}\right) .
\end{aligned}
$$

Therefore, $T(f)=\phi_{1}(f) T\left(e^{\sharp}\right)$. This follows that for each $f \in A$ and $T \in$ ${ }_{\phi_{1}} B\left(A^{\sharp}, I_{1}\right)$ we have

$$
\begin{aligned}
\rho(f \cdot T) & =(f \cdot T)\left(e^{\sharp}\right)=T\left(e^{\sharp} f\right)=T(f) \\
& =\phi_{1}(f) T\left(e^{\sharp}\right)=f \cdot T\left(e^{\sharp}\right)=f \cdot \rho(T) .
\end{aligned}
$$

Hence, $\rho$ is a left $A$-module morphism which is also a left inverse for ${ }_{\phi_{1}} \Pi^{\sharp}$. Therefore, $\ell^{1}\left(\mathbb{N}_{\wedge}\right)$ is $\phi_{1}$-injective as a Banach left $\ell^{1}\left(\mathbb{N}_{\wedge}\right)$-module.

Case 2: We prove that $\ell^{1}\left(\mathbb{N}_{\wedge}\right)$ is $\phi_{n}$-injective as a Banach left $\ell^{1}\left(\mathbb{N}_{\wedge}\right)$-module for each $n \geq 2$. Since $I_{n} \in \mathbf{I}_{n}$ - $\bmod$ has the identity $\delta_{n}$, so it is faithful. By Theorem 2.6, it suffices to show that $I_{n} \in \mathbf{I}_{n}$ - $\mathbf{m o d}$ is $\left(\phi_{n}\right)_{\mid I_{n}}$-injective. Let $\widehat{\phi_{n}}$ be the semi-character on $\mathbb{N}_{\wedge}$ associated with $\phi_{n}$. Since $S=(\{1,2,3, \ldots, n\}, \wedge)$ is a semilattice and $\widehat{\phi_{n}}(1)=0$, it follows from [4, Theorem 2.1] that $I_{n}=$ $\ell^{1}(\{1,2,3, \ldots, n\}, \wedge)$ is $\left(\phi_{n}\right)_{\mid I_{n}}$-amenable. Therefore, we conclude that $c_{0}(S)$ is $\left(\phi_{n}\right)_{\mid I_{n}}$-flat in $\bmod -\mathbf{I}_{n}$ by [10, Proposition 3.1]. Hence, $I_{n}=c_{0}(S)^{*}$ is $\left(\phi_{n}\right)_{\mid I_{n}{ }^{-}}$ injective in $\mathbf{I}_{n}$-mod and the proof is complete.

Let $\ell^{1}\left(\mathbb{N}_{\vee}\right)$ be the semigroup algebra on semigroup $S=(\mathbb{N}, \vee)$ with the following product:

$$
\mathbb{N} \times \mathbb{N} \longrightarrow \mathbb{N}, \quad(m, n) \longrightarrow m \vee n=\max \{m, n\} .
$$

It is easy to check that $\Phi_{S}=\left\{\widehat{\psi}_{n}: n \in \mathbb{N}\right\} \cup\left\{\widehat{\psi_{S}}\right\}$, where $\widehat{\psi_{S}}$ is the augmentation character and for each $n \in \mathbb{N}$,

$$
\widehat{\psi}_{n}(m)=\left\{\begin{array}{ll}
1 & \text { if } \quad m \leq n \\
0 & \text { if } \quad m>n
\end{array} \quad(m \in \mathbb{N}) .\right.
$$

In [11, Example 5.6], it is proved that $\ell^{1}\left(\mathbb{N}_{\vee}\right)$ is not injective as a Banach left $\ell^{1}\left(\mathbb{N}_{\vee}\right)$-module. In the following theorem, we show that $\ell^{1}\left(\mathbb{N}_{\vee}\right)$ is $\phi$-injective for each $\phi \in \Delta\left(\ell^{1}\left(\mathbb{N}_{\vee}\right)\right)$.

Theorem 3.3. $\ell^{1}\left(\mathbb{N}_{\vee}\right)$ as a Banach left $\ell^{1}\left(\mathbb{N}_{\vee}\right)$-module is $\phi$-injective for each $\phi \in \Delta\left(\ell^{1}\left(\mathbb{N}_{\vee}\right)\right)$.

Proof. By [4, Corollary 2.2], it follows that $\ell^{1}\left(\mathbb{N}_{\vee}\right)$ is character amenable. Hence, for each $\phi \in \Delta\left(\ell^{1}\left(\mathbb{N}_{\vee}\right)\right), \ell^{1}\left(\mathbb{N}_{\vee}\right)$ is $\phi$-amenable. On the other hand, since $\mathbb{N}_{\vee}$ is weakly cancellative by $[2$, Theorem 4.6$]$, we conclude that $c_{0}\left(\mathbb{N}_{V}\right)$ is a Banach $\ell^{1}\left(\mathbb{N}_{\vee}\right)$-module. Hence, $c_{0}\left(\mathbb{N}_{\vee}\right)$ is $\phi$-flat as a Banach right $\ell^{1}\left(\mathbb{N}_{\vee}\right)$-module [10, Proposition 3.1]. This follows that $c_{0}\left(\mathbb{N}_{\vee}\right)^{*}=\ell^{1}\left(\mathbb{N}_{\vee}\right)$ is $\phi$-injective as a Banach left $\ell^{1}\left(\mathbb{N}_{\vee}\right)$-module. 
For each $n \in \mathbb{N}$, let $J_{n}$ be the closed ideal of $A=\ell^{1}\left(\mathbb{N}_{\vee}\right)$ generated by $\left\{\delta_{n}, \delta_{n+1}, \ldots\right\}$. It is easy to see that $J_{n}$ is invariantly complemented in $\ell^{1}\left(\mathbb{N}_{\vee}\right)$. Indeed, it is sufficient to consider the map $Q_{n}: A \longrightarrow J_{n}$ defined by

$$
Q_{n}(f)=\left(\sum_{i=1}^{n} f(i)\right) \delta_{n}+\sum_{i=n+1}^{\infty} f(i) \delta_{i} \quad(f \in A) .
$$

It is straightforward to check that $Q_{n}$ is an onto projection in ${ }_{A} B\left(A, J_{n}\right)$.

As a consequence of Corollary 2.11 and Theorem 3.3, we give the following result.

Corollary 3.4. For each $n \in \mathbb{N}$, $J_{n}$ as a Banach left $\ell^{1}\left(\mathbb{N}_{\vee}\right)$-module is $\phi$-injective for each $\phi \in \Delta\left(\ell^{1}\left(\mathbb{N}_{\vee}\right)\right)$ with $\phi_{\mid J_{n}} \neq 0$.

\section{REFERENCES}

1. H. G. Dales, Banach algebras and automatic continuity, Clarendon press, Oxford, 2000.

2. H. G. Dales, A. T. Lau and D. Strauss, Banach algebras on semigroups and their compactifications, Memoirs of American Math. Soc. 205 (2010), 1-165.

3. H. G. Dales, M. E. Polyakov, Homological properties of modules over group algebras, Proc. London Math. Soc. 89 (2004), 390-426.

4. M. Essmaili, M. Filali, $\phi$-amenability and character amenability of some classes of Banach algebras, Houston J. Math. Vol 39. No 2 (2013), 515-529.

5. B. E. Forrest, Amenability and bounded approximate identities in ideals of $A(G)$, Illinois J. Math. Vol 34. No 1 (1990), 1-25.

6. A. Ya. Helemskii, A certain class of flat Banach modules and its applications, Vestnik. Moskov. Univ. Ser. Mat. Mekh. 27 (1972), 29-36.

7. E. Kaniuth, A. T. Lau and J. S. Pym, On $\varphi$-amenability of Banach algebras, Math. Proc. Cambridge philos. Soc. 144 (2008), 85-96.

8. E. Kaniuth, A. T. Lau and J. S. Pym, On character amenability of Banach algebras, J. Math. Anal. Appl. 344 (2008), 942-955.

9. M. S. Monfared, Character amenability of Banach algebras, Math. Proc. Cambridge Philos. Soc. 144 (2008), 697-706.

10. R. Nasr-Isfahani, S. Soltani Renani, Character injectivity and projectivity of Banach modules, Quart. J. Math. (2013), Doi: 10.1093/qmath/hat015.

11. P. Ramsden, Homological properties of modules over semigroup algebras, J. Funct. Anal. 258 (2010) 3988-4009.

12. P. Ramsden, Homological properties of semigroup algebras, Ph. D. Thesis, University of Leeds, 2008.

13. M. C. White, Injective modules for uniform algebras, Proc. London Math. Soc. (3) 73 (1996), 155-184.

Faculty of Mathematical and Computer Science, Kharazmi University, 50 Taleghani Avenue, 15618 Tehran, Iran.

E-mail address: m.essmaili@khu.ac.ir

E-mail address: fozouni@khu.ac.ir

E-mail address: laali@khu.ac.ir 